[ED] EBERLEIN,P.

"When is a Geodesic Flow of Anosov Type? I.", $J$. Diff. Geom. 8 (1973) 437-463.

[Es] ESCHENBURG, J.

"Horospheres and the Stable Part of the Geodesic Flow". Math.Z. 153 (1977) 237-251.

[H] HEDLUND, G.A.

"The Dynamics of Geodesic Flows", Bull. Amer. Math. Soc. 45 (1939) $241-260$.

$[H+H]$ HEINTZE, E, and HOF, H.Ch. Im.

"Geometry of Horospheres", J. Diff.Geom., 12 (1977) 481491.

[K] KLINGENBERG, W。

"Riemannian manifolds with Geodesic Flow of Anosov Type", Ann. of Math., 99 (1974) 1-13.

[P] PESIN, $Y a$. B.

"Characteristic Lyapunov Exponents and Smooth Ergodic Theory", Russian Math. Surveys, 32 (1977) 55-114.

Mathematics Department.

University College.

Conk.

\section{REMARKS ON 'AN ELEMENTARY NUMBER THEORY RESULT'}

\section{David Singmaster}

In a joint note published in this Newsletter (No. 12, December 1984, pp. 10-13), Peter Birch and I showed that $\phi(n)>n / \log n$ except for $n=1,2,3,4,6,10,12,18$ or 30 . For convenience, let us set $\phi(n)=n^{-1} \phi(n) \log n$, so the above says $\Phi(n)>1$, except for the values given. Our proof used Bertrand's postulate, so it was not entirely elementary. I have just found that Alan Baker gives an entirely elementary proof that $\phi(n)>\frac{1}{4}$ for $n>1[1, p \cdot 12]$. Further care with his argument shows the asymptotic result $\Phi(n)>\frac{1}{2}-E$ for all large enough $n$ and explicit calculation would show $\Phi(n)>2 / 5$ for all $n>2$.

Baker's argument, in more detail, is as follows. First consider $\sigma(n)$, the sum of the divisors of $n$. Then

$$
\begin{aligned}
& \sigma(n)=\Sigma_{d \mid n^{d}}=\Sigma_{d \mid n^{n}} n / d=n \Sigma_{d \mid n^{1 / d} \leqq n \Sigma_{d \mid n}} d^{-1} \text {, so } \\
& \sigma(n) \leqq n(1+\log n) .
\end{aligned}
$$

Consider now $f(n)=\sigma(n) \phi(n) n^{-2}$. This is multiplicative and $f\left(p^{j}\right)=1-p^{-j-1}$. Then

$$
\begin{gathered}
f(n)=\pi_{p} j_{\mid n}\left(1-p^{-j-1}\right) \geqq \pi_{p} j_{\leqq n}\left(1-p^{-j-1}\right) \geqq \pi_{p^{2} \leqq n}\left(1-p^{-2}\right) . \\
\geqq \pi_{1 \leqq m^{2} \leqq n}\left(1-m^{-2}\right)=\frac{1}{2}\left(1+[\sqrt{n}]^{-1}\right), \text { for } n \geqq 4 \text {, so that } \\
\sigma(n) \phi(n) n^{-2} \geqq \frac{1}{2}\left(1+n^{-\frac{1}{2}}\right),
\end{gathered}
$$

and this is seen to hold for $n \geqq 3$.

From (1) and (2), we have

$$
\phi(n) \geqq \frac{1}{2} n\left(1+n^{-\frac{1}{2}}\right)(1+\log n)^{-1} \text {, for } n \geqq 3 \text {. }
$$


Baker's argument takes the simpler result $f(n) \geq \frac{1}{2}$ instead of (2) and then uses $1+\log n<2 \log n$ for $n>1$ to deduce $\Phi(n)>t$ But (3) clearly gives the asymptotic result $\Phi(n)>\frac{1}{2}-E$, for large enough $n$. Explicit calculation of the ratio of $n / \log n$ to $\frac{1}{2} n\left(1+n^{-\frac{1}{2}}\right)(1+\log n)^{-1}$ gives a ratio of the $n=1$, of 349 for $n=2$ and a ratio $\geqq .412$ for $n>2$, so this elementary method yields $\phi(n)>2 / 5$ for $n>2$. . Further calculation, based on our result, shows that we actually have $\Phi(n) \geq(\log 6) / 3=.59725$ for $n>2$.

\section{REFERENCE}

1. BAKER, A

'A Concise Introduction to the Theory of Numbers', (Cambridge University Press, Cambridge, 1984).

Polytechnic of the South Bank, London, SEI OAA

\section{MATHEMATICAL EDUCATION}

REPORT ON THE BASIC MATHEMATICAL SKILLS TEST OF FIRST YEAR STUDENTS IN CORK RTC IN 1984

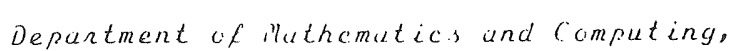

\section{INTRODUCTION}

A test was given to all first year students in Dctober 1984 to assess their basic mathematical competency. ults of this test show that our student intake have fundamental deficiencies in their basic mathematics.

While the direct remedy of this situation is outside our control, it is hoped that those involved in the teaching and drawing up of mathematical syllabi at primary and secondary level will consider the implications o: this report.

\section{THE TEST: ITS CONSTRUCTION AND PURPOSE}

A copy of the test paper is given in Appendix A. It consists of 20 questions which the students had to attempt without the aid of tables or calculators in the allatted time of one hour. The aim of the questions and acceptable answers are also given in Appendix A.

After careful consideration as to what basic mathematical skills students should have after completing their Leaving Certificate (L/C) the pass level for this test was set at 15 or more correct answers.

As well as the answers to the test questions, the sex and best L/C mathematics grade of the students were recorded. 